\title{
Percepção dos estudantes de terapia ocupacional sobre a interprofissionalidade: influências na formação acadêmica
}

\author{
Maria Luiza Rodrigues Torres \\ Membro do grupo de Pesquisa "Ensino, Saúde e Sociedade" - UNCISAL/CNPq \\ Graduação em Terapia Ocupacional - UNCISAL \\ $\triangle$ luizatorres18@gmail.com \\ Kássia Luzia Lima Rodrigues \\ Membro do grupo de Pesquisa "Ensino, Saúde e Sociedade" - UNCISAL/CNPq \\ Graduação em Terapia Ocupacional - UNCISAL \\ $\bowtie$ kassialuziar@gmail.com \\ Claudio José dos Santos Júnior \\ Membro do grupo de Pesquisa "Ensino, Saúde e Sociedade" - UNCISAL/CNPq \\ Mestrando em Ensino na Saúde e Tecnologia - UNCISAL \\ Graduação em Ciências Biológicas - ULBRA \\ Graduando em Medicina - UNCISAL \\ $\square \underline{\text { claudiosantos_al@hotmail.com }}$ \\ John Victor dos Santos Silva \\ Membro do grupo de Pesquisa "Ensino, Saúde e Sociedade" - UNCISAL/CNPq \\ Mestrando em Enfermagem Psiquiátrica pela Escola de Enfermagem de Ribeirão Preto - EERP/USP \\ Graduação em Enfermagem - UNCISAL \\ 凶john.setedejulho@gmail.com
}

\section{Mara Cristina Ribeiro}

Pró-reitora de Pesquisa e Pós-graduação da Universidade Estadual de Ciências da Saúde de Alagoas - UNCISAL Docente Titular da Universidade Estadual de Ciências da Saúde de Alagoas - UNCISAL

Docente do Mestrado em Pesquisa em Saúde - Centro Universitário Cesmac

Líder do grupo de Pesquisa "Ensino, Saúde e Sociedade" - UNCISAL/CNPq

Doutora em Ciências - USP

Mestre em Enfermagem Psiquiátrica - USP

Especialista em Saúde Pública - USP

Graduação em Terapia Ocupacional - USP

$\triangle$ mara.uncisal@yahoo.com.br

Recebido em 16 de agosto de 2020

Aceito em 18 de maio de 2021

\section{Resumo:}

O ensino integrado e interprofissional vêm sendo aplicado como estratégia na formação dos estudantes da área da saúde. Assim, este estudo teve como objetivo conhecer como o estudante de Terapia Ocupacional percebe sua formação integrada e interprofissional aos outros cursos da saúde. Trata-se de pesquisa qualitativa com uso de entrevistas analisadas pela técnica de Análise de Conteúdo. Foram entrevistados estudantes do curso de Terapia Ocupacional de uma universidade pública do nordeste brasileiro. $O$ estudo evidencia a dificuldade dos discentes em discorrer sobre os

\footnotetext{
${ }^{1}$ Artigo derivado de Trabalho de Conclusão de Curso apresentado por Maria Luiza Rodrigues Torres para obtenção de título de Graduação em Terapia Ocupacional pela Universidade Estadual de Ciências da Saúde de Alagoas no ano de 2017 e orientação da Profa. Dra Mara Cristina Ribeiro.
} 
conceitos de formação interprofissional e trabalho em equipe; também aponta que o acesso às práticas interprofissionais é pequeno, principalmente nas atividades curriculares, demonstra, portanto, que para ter experiências desse tipo na formação é necessário o envolvimento em atividades de extensão ou extracurriculares. Conclui-se que a Universidade deve estimular a formação interprofissional por meio da qualificação dos docentes, integração das grades curriculares dos diversos cursos, e incentivo às metodologias de ensino que intensifiquem as práticas interprofissionais.

Palavras-chave: Terapia Ocupacional, Educação Interprofissional, Educação Superior, Pesquisa qualitativa..

\title{
Occupational therapy students' perception of interprofessionality: influences on academic training
}

\begin{abstract}
:
Integrated and interprofessional education has been applied as a strategy in the training of students in the health field. Thus, this study aimed to understand how the Occupational Therapy student perceives his integrated and interprofessional training to other health courses. It is a qualitative research using interviews analyzed by the Content Analysis technique. Students from the Occupational Therapy course at a public university in northeastern Brazil were interviewed. The study highlights the students' difficulty in discussing the concepts of interprofessional training and teamwork; it also points out that access to interprofessional practices is small, especially in curricular activities, demonstrating, therefore, that to have experiences of this type in training, it is necessary to be involved in extension or extracurricular activities. It is concluded that the University must stimulate the interprofessional training through the qualification of the teachers, integration of the curricular grids of the different courses, and incentive to the teaching methodologies that intensify the interprofessional practices.
\end{abstract}

Keywords: Occupational therapy, Interprofessional Education, College education, Qualitative research.

\section{Percepción de la interprofesionalidad de los estudiantes de terapia ocupacional: influencias en la formación académica}

\section{Resumen:}

La educación integrada e interprofesional se ha aplicado como estrategia en la formación de estudiantes en el campo de la salud. Así, este estudio tuvo como objetivo comprender cómo el estudiante de Terapia Ocupacional percibe su formación integrada e interprofesional a otros cursos de salud. Es una investigación cualitativa que utiliza entrevistas analizadas mediante la técnica de Análisis de contenido. Se entrevistó a estudiantes del curso de Terapia Ocupacional de una universidad pública del noreste de Brasil. El estudio destaca la dificultad de los estudiantes para discutir los conceptos de formación interprofesional y trabajo en equipo; También señala que el acceso a las prácticas interprofesionales es reducido, especialmente en las actividades curriculares, demostrando, por tanto, que para tener experiencias de este tipo en la formación, es necesario involucrarse en actividades de extensión o extraescolares. Se concluye que la Universidad debe estimular la formación interprofesional a través de la calificación de los docentes, la integración de las cuadrículas curriculares de los diferentes cursos, y el incentivo a las metodologías de enseñanza que intensifiquen las prácticas interprofesionales.

Palabras clave: Terapia ocupacional, Educación interprofesional, Educación universitária, Investigación cualitativa. 


\section{INTRODUÇÃO}

A Terapia Ocupacional é uma profissão de nível superior da área da saúde, que pode atuar no Sistema Único de Saúde (SUS), no Sistema Único da Assistência Social (SUAS), na Educação e na Cultura (CAMARGOS et al, 2017), de forma a garantir a integralidade da assistência em todos os seus níveis de complexidade. Sua prática é marcada por diversos modelos e técnicas de intervenção em decorrência das diferentes concepções de homem, saúde, doença e atividades, assumidas concomitantemente pelas ciências que a embasam (MEDEROS, 2009). Interessa-se pela problemática do homem e se relaciona com o mesmo num campo de complexidades no qual exige estudos e conhecimentos interdisciplinares. Por isso, sua formação necessita de várias ciências inter-relacionadas para atender as diversas demandas trazidas (SOARES, 2007).

Assim, a Terapia Ocupacional em sua origem e desenvolvimento, enquanto formação e atuação profissional, ao integrar-se com objetos de conhecimento de outras ciências, diferencia-se destas ao tomar como ponto de partida essa integração para o estabelecimento de sua intervenção e da produção do seu conhecimento (DRUMMOND, 2007).

Em 2002, buscando integrar a formação dos profissionais da saúde, foram aprovadas as Diretrizes Curriculares Nacionais (DCN) dos cursos de graduação em saúde, incluindo o Curso de Terapia Ocupacional. No entanto, ainda existem diversas lacunas na formação que mostram a necessidade de melhor capacitação nas Escolas de Nível Superior para garantir um ensino capaz de integrar as profissões. (LINS; MATSUKURA, 2015).

Em estudo realizado por Ceccim e Feuerwerker (2004) que teve como objetivo formular uma teoria-caixa de ferramentas que permitisse a análise crítica da educação no setor da saúde detectou-se que a área de maior defasagem dentro do Sistema Único de Saúde é a formação. Observa-se que, com base na integralidade da saúde é necessário currículo que assegure que esse princípio básico seja implementado na formação de seus estudantes. Desta forma, os autores destacam que “[...] a integralidade da atenção envolve a compreensão da noção de ampliação da clínica, o conhecimento sobre a realidade, o trabalho em equipe multiprofissional e transdisciplinar e a ação intersetorial" (CECCIM; FEUERWERKER, 2004, p.51). 
Conforme Ferreira (2017), a formação interprofissional trata-se de uma forma de educação onde há compartilhamento entre as profissões, estímulo para que os estudantes de diferentes cursos de determinada área (como a saúde) aprendam com e sobre as outras profissões. Esse estímulo também favorece o trabalho em equipe, a responsabilidade, a criatividade e outras competências e habilidades necessárias para o trabalho nos serviços de saúde (SILVA; SANTOS JÚNIOR; RIBEIRO, 2019).

Tendo como foco a formação em terapia ocupacional em uma universidade pública do nordeste, que oferta diferentes cursos superiores na área da saúde, entre eles: Medicina, Fonoaudiologia, Enfermagem e Fisioterapia, com os quais a Terapia Ocupacional se relaciona no campo profissional, este estudo se propôs a pesquisar como o estudante de Terapia Ocupacional percebe sua formação integrada aos outros cursos da saúde, visando construir reflexão critica, formular propostas, sugerir e redimensionar estratégias já utilizadas, para a melhor formação profissional na perspectiva da integralidade do cuidado.

\section{MATERIAL E MÉTODOS}

O presente estudo faz parte de pesquisa mais ampla, denominada "Terapia Ocupacional e interprofissionalidade: radiografia das bases integrativas da formação e atuação profissional", desenvolvida no Grupo de Pesquisa Ensino, Saúde e Sociedade, de abordagem qualitativa, caracterizada como exploratória e descritiva.

A utilização da metodologia qualitativa nesse trabalho justifica-se, pois, conforme Minayo (2010) indica, por meio dela pode-se trabalhar com o universo dos significados, motivos, aspirações, crenças, valores e atitudes.

A investigação se desenvolveu nas dependências de uma universidade estadual de uma capital do nordeste brasileiro que tem entre os seus objetivos favorecer atividades integradas de ensino, pesquisa, extensão e assistência na área da Saúde. 
Atualmente a Universidade passa por uma reformulação dos Projetos Pedagógicos dos Cursos, incluindo eixos integradores às matrizes curriculares de todos os cursos de bacharelado, exceto Medicina e Enfermagem.

Nesse contexto, o curso de Terapia Ocupacional está dividido em dois diferentes grupos, aquele que representa os estudantes que já estão no último ano e, que, portanto, não tiveram em seus currículos as disciplinas e módulos integrados e, aquele que representa os estudantes que ingressaram na Universidade depois da reformulação curricular e, por isso, já estão vivenciando essa integralização em sua formação.

Foram contatados 24 acadêmicos do curso de terapia Ocupacional. Destes, apenas 12 aceitaram participar do estudo. 0 critério de inclusão foi estar no último ano do curso e de exclusão os que não estivessem no novo modelo curricular.

O período para a realização das entrevistas foi de outubro a dezembro de 2016. Os estudantes foram contatados por meio da técnica de busca ativa, na própria estrutura da universidade e por meio de redes sociais. Aos que se mostraram interessados em participar, foram agendados encontros para a realização de entrevista individual norteada por um formulário semiestruturado. Com a permissão dos participantes, as entrevistas foram gravadas exceto uma, que foi registrada a entrevista por meio de diário de campo, por ter sido recusada a gravação.

Todo o material produzido foi transcrito em sua íntegra e analisado sob a luz da técnica de Análise de Conteúdo, na modalidade categorial, seguindo as fases fundamentais: de pré-análise e exploração de todo o material transcrito das entrevistas, em que foram observadas as operações de codificação, decomposição e recomposição (BARDIN, 2011).

Este estudo respeitou todos os preceitos éticos estabelecidos pela resolução no 510/2016/CNS, sendo apreciado pelo Comitê de Ética em Pesquisa (CEP) da instituição proponente, sendo aprovado pelo parecer $n^{\circ}$ 1.578.952. Aos estudantes foram apresentados os objetivos da pesquisa, sendo coletada a autorização de participação por meio do Termo de Consentimento Livre e Esclarecido (TCLE). Para garantir o sigilo e anonimato dos participantes, utilizou-se o código "entrevistado" para todos os estudantes, seguido de um número de 1 a 12, distribuindo aleatoriamente. 


\section{RESULTADOS E DISCUSSÃO}

A partir das análises das falas dos entrevistados, emergiram as seguintes categorias: Conceituação de trabalho em equipe e interprofissionalidade; Participação em módulos ou disciplinas com estudantes de outras áreas ou profissões; Participação em Extensões Universitárias; e Estímulo por parte da Universidade.

\section{Conceituação de trabalho em equipe e interprofissionalidade}

Sobre o que os acadêmicos compreendem por atuação interprofissional, as respostas obtidas variaram conforme presença no estágio de saúde coletiva e suas experiências extracurriculares, portanto, foi possível identificar que quanto maior a participação em disciplinas compostas por diferentes formações ou mais ações extracurriculares, no que tange a interprofissionalidade, maior a segurança e conhecimento do discente para abordar a temática.

[...] é pra o bem-estar do paciente, é em conjunto, a gente não vai mais assim, num plano cartesiano, ah, vamos cuidar hoje da perna, do braço, não. A gente vê o paciente como um todo, elabora o objetivo que a gente quer traçar e, tipo, lá no estágio somos 3, Fono, Fisio e T.O. e a gente atende em conjunto mesmo, não especifica ali, o que cada um vai fazer, é uma junção (Entrevistado 03).

Alguns esclarecimentos se fazem necessários para que possamos entender o contexto das respostas: Além daqueles que não tiveram nenhuma prática interprofissional, também pode-se observar que outros, como o entrevistado 03 (da fala acima), se refere à experiência obtida em um dos campos de estágio, muito mencionado em todo levantamento de dados com outros estudantes de Terapia Ocupacional, que foi o estágio em Saúde Coletiva.

As ações desenvolvidas neste estágio são feitas de forma interprofissional e tudo é planejado em conjunto, desde as reuniões para a elaboração de possíveis atividades até a execução das ações propriamente ditas. O estágio é desenvolvido por uma equipe composta por professores/preceptores de formação em terapia ocupacional, fonoaudiologia, fisioterapia e os estudantes das respectivas áreas. No entanto, nem todos os estudantes 
tiveram a oportunidade de vivenciar essa prática, apenas 8 estagiários de uma turma de 24 estudantes.

As ações desse estágio acontecem em atendimento domiciliar, com um grupo de mulheres, um grupo de pescadores e em uma creche, tudo no bairro próximo da Universidade. Considerando que a interprofissionalidade é uma forma de trabalho em equipe, na qual busca-se refletir sobre os papéis profissionais almejando dar resolutividade por meio da negociação na tomada de decisões, construindo conhecimentos e respeitando as singularidades das diferentes práticas profissionais, reduzindo custos e ampliando o cuidado (ARAÚJO et al., 2017) algumas destas características também foram indicadas pelos estudantes que participaram do estudo.

Quando questionados sobre o que seria trabalho em equipe, alguns confundiram com o conceito de interprofissionalidade, como se os conceitos tivessem o mesmo significado, ou seja, fossem sinônimos:

É [...], Trabalho em equipe é importante porque a gente não consegue fazer nada sozinho, a gente tem a nossa área, né? Mas em alguns momentos precisa do saber do outro, outras áreas. A importância é essa, a união de saberes. Nessa questão também, interprofissional, que traz saberes de todas as áreas pra o bem dos indivíduos que serão assistidos (Entrevistado 03).

$\mathrm{Na}$ interprofissionalidade cada um coopera com seu conhecimento. Trabalhar em equipe é agregar todos os conhecimentos (Entrevistado 06).

A interprofissionalidade, assim como a multiprofissionalidade, são formas de trabalho em equipe, em ambas se trabalha em grupo, com relação recíproca entre as intervenções e interação (UCHÔA, 2012). Logo, compreende-se que nem sempre um trabalho em equipe é interprofissional, característica essa mencionada em alguns dos relatos, mas confundida em outros. Para melhor compreender o trabalho interprofissional, deve-se diferenciar a particularidade das diferentes formas de trabalho em equipe.

A multiprofissionalidade, apesar de se constituir como um trabalho em equipe, indica a ação de várias profissões, mas não necessariamente, implica na interrelação da atuação dessas diferentes profissões. Já a interprofissionalidade deve corresponder à 
atuação de diferentes formações profissionais em ações conjuntas, integradas e interrelacionadas (SILVA; RIBEIRO, 2018).

A interrelação que é mediada por preceptores, em um espaço de aprendizado com objetivos de formação integrada em saúde, deve se caracterizar como interprofissional, propondo dinâmicas de trabalho colaborativo e aprendizado mútuo, propiciando a transformação das práticas e o desenvolvimento de competências comuns. (ARRUDA et al., 2018)

Portanto, investir nesses espaços de formação integrada enriquece o processo de aprendizados dos estudantes, proporcionando que os mesmo possam estar aptos para o trabalho em equipe de saúde com diversas categorias profissionais.

\section{Participação em módulos ou disciplinas com estudantes de outras áreas ou profissões}

Sobre a questão dos discentes terem frequentado módulos ou disciplinas durante a graduação, em conjunto com estudantes de outras áreas ou profissões, pode-se dividir os participantes do estudo em dois grupos: os que tiveram essa experiência e os que não tiveram.

Segundo os entrevistados, o acesso às práticas interprofissionais foi limitado aos estudantes que participaram do estágio de Saúde Coletiva, aspecto esse que dificulta ao aluno contemplar as competências gerais previstas como necessárias na formação dos profissionais de saúde segundo as Diretrizes Curriculares Nacionais (DCN), como a atenção à saúde, o princípio da integralidade, a tomada de decisões, a comunicação, a liderança, a administração, o gerenciamento e a educação permanente (COLLISELLI et al., 2009).

Dessa forma, entre os estudantes que participaram de disciplinas com outras áreas ou profissões, a grande maioria só teve esse contato ao fim do curso, no estágio propriamente dito, limitando-se ainda a apenas alguns campos de atuação. Não há consenso em quando se deve iniciar a formação interprofissional (COLLISELLI et al., 2009), ela pode se dar ao início do curso ou no estágio, mas é preciso apontar que os discentes estão abertos à possibilidade deste tipo de aprendizagem e seus pormenores podem ser trabalhados ao longo do curso, favorecendo a formação de profissionais mais competentes 
e preocupados com as necessidades do sistema de saúde e da qualidade do atendimento de seus usuários (HORSBURGH; LAMDIM; WILLIAMSON, 2001).

Foram mencionadas outras experiências, como a observação da equipe interprofissional do Núcleo de Saúde da Família - NASF assim como alunos que tiveram oportunidade de cursar a disciplina optativa "Educação para relações étnico-raciais e afirmação das diferenças", compartilhando aprendizagem com alunos de medicina e de fisioterapia. No entanto, a mesma não ofereceu experiência prática, apenas teórica.

Os entrevistados relatam que a experiência em conjunto com estudantes ou profissionais de outras áreas foi positiva, contribuindo para melhorar as discussões dos casos, ampliar o olhar na perspectiva do cuidado, para maior conhecimento sobre as diversas profissões em compartilhamento, na troca de conhecimento, no aprendizado e para facilitar o futuro profissional. Tais pontos corroboram com os diversos pontos elencados por Silva, Scapin e Batista (2011), como o favorecimento da experiência interprofissional para a desconstrução de atitudes e percepções negativas entre os profissionais de saúde.

Sobre as dificuldades encontradas na relação e no aprendizado com estudantes de outros cursos, foram citados de forma mais frequente os problemas de ordem interpessoal, como dificuldade em lidar com o outro assim como para aceitar a forma de agir de alguns profissionais ou estudantes que não compreendiam a atuação interprofissional. Um dos entrevistados relatou que se sentiu mal por estar em minoria numa turma com discentes de vários cursos, em sua maioria de medicina.

Senti um pouco de dificuldade por "eles" (se referindo aos estudantes de medicina) serem uma maioria e assim de terapeuta ocupacional só tinha eu [...], mas assim, nada que não superasse os desafios, as dificuldades, eu sei que em tudo, no fim dava certo, nos uníamos, mas assim, sempre essa dificuldade por questão de curso (Entrevistado 09).

Por meio do grupo focal realizado com estudantes de Medicina, com intuito de debater sobre a formação de profissionais da Saúde e sua adequação à visão ampliada de saúde, colocada pelo SUS na Estratégia de Saúde da Família - ESF, Moretti-Pires (2009) identificou que para os alunos, prevalece ainda, a visão do médico como superior aos 
demais profissionais, que o trabalho deve ser centralizado no saber médico e justificam esse pensamento pelo grande aporte teórico da formação, sugerindo ser maior que o de outras formações da saúde. Essa visão é contrária à perspectiva baseada nos princípios do SUS, demonstrando um ponto de vista reducionista e fragmentário no que se refere ao trabalho em equipe.

Segundo os entrevistados, essa dificuldade com os estudantes do curso de medicina também foi sentida, mas na maior parte das vezes superada. Os discentes de Terapia Ocupacional participantes da pesquisa indicaram que melhor relacionavam-se com os estudantes de Fonoaudiologia e de Fisioterapia e citam que, de maneira informal, os casos clínicos eram discutidos com maior frequência entre essas áreas e, com menor frequência, essas trocas de informação sobre os usuários aconteciam com os alunos de Enfermagem e, ainda mais raramente, com os de Medicina.

No geral, para os estudantes que tiveram oportunidade de vivenciar essas práticas, lhes foi possibilitada a construção de diversas competências para a vida profissional, como uma compreensão integral sobre o usuário, um aprendizado mais efetivo sobre a importância da construção conjunta de práticas entre as diversas profissões e um reconhecimento da necessidade de diálogos interprofissionais na busca de objetivos em comum.

Os discentes que não cursaram módulos ou disciplinas integradas, apesar de não terem tido oportunidade de conhecer a prática interprofissional, comentam sobre os colegas que tiveram essa oportunidade e reconhecem que seria de grande importância viver essa experiência e mencionam as habilidades que poderiam ter desenvolvido.

As habilidades seriam, é, de saber trabalhar em equipe, de saber ouvir o outro, de saber questionar a melhor maneira, a forma de intervir [...] lidar com esses profissionais, saber se comportar numa equipe, definir estratégias que sejam positivas pra equipe, pensando naquele usuário, no paciente, e então é mais questão disso, de respeito, de lidar com o outro, criar estratégias em conjunto, são mais essas habilidades que a gente consegue adquirir no trabalho em equipe, e sozinho é mais o seu pensamento, sua forma de intervir e no conjunto você precisa compartilhar esses saberes (Entrevistado 04). 
Dentre as profissões citadas pelos entrevistados como mais importantes para trabalhar de forma integrada, mencionaram-se todas da área da saúde assim como Psicologia, Pedagogia e a Assistência Social.

A formação em saúde deve continuamente considerar que os profissionais necessitarão trabalhar juntos. Isso torna evidente a necessidade de modificar a formação como um todo, utilizando metodologia pautada em modelo que busque construir formação e prática profissional que possibilite integrar a formação em saúde à realidade dos serviços prestados à comunidade, de forma que englobe todos os profissionais necessários às equipes (SILVA; SCAPIN; BATISTA, 2011; SILVA, SANTOS JÚNIOR; RIBEIRO, 2019).

Entendendo que a formação em saúde deve acontecer continuamente ao longo da graduação, o ensino e as práticas integradas também devem ocorrer em diferentes momentos do curso e não apenas em disciplinas iniciais ou pontuais.

\section{Participação em Extensões Universitárias}

Todos os entrevistados tiveram experiências extracurriculares durante a graduação, seja por meio das ligas acadêmicas, da participação em congressos, projetos de extensão e até mesmo diretórios acadêmicos, que propiciaram experiências e contato com diferentes profissões e estudantes de outras áreas.

Essas atividades são práticas que complementam o currículo, assim como ampliam o conhecimento, componentes enriquecedores e implementadores do próprio perfil do aluno, demonstrando que a vivência resulta numa maior compreensão do trabalho em equipe e papel profissional das áreas com as quais o aluno se relaciona. (PERES; ANDRADE; GARCIA, 2007).

A entrevistada que menciona maior contato com alunos de todos os cursos relata que essa facilidade se dá em decorrência da sua participação no Diretório Central dos Estudantes - DCE:

[...] É, eu tenho contato com estudantes de todos os cursos daqui, principalmente por causa do diretório, né? Que é o Diretório Central dos Estudantes, aí eu tenho 
contato com os meninos da medicina, da fonoaudiologia, da enfermagem, da fisio e é um contato assim, do dia a dia, de ver na universidade e conversar (Entrevistada 05).

Dentre as ações extensionistas, foi citado o Projeto Sorriso de Plantão, projeto formalizado pela parceria de uma Universidade Federal com uma Universidade Estadual que acontece de forma multidisciplinar e participam graduandos de todos os cursos (denominados palhaços doutores) com intuito de promover a humanização dentro de cinco hospitais em suas alas pediátricas (TORRES et al., 2016), também foram mencionadas a participação em diversas ligas acadêmicas, o Programa de Educação pelo Trabalho para a Saúde (PET-Saúde) e o Projeto Rondon.

Para a educação superior em saúde alcançar a formação de profissionais que atendam e trabalhem dentro da perspectiva dos preceitos do SUS, foram propostas as Diretrizes Curriculares Nacionais (DCN), que visam, dentre outras coisas, desenvolver atividades de caráter interprofissional que impulsionem a formação integrada de ensinoserviço-comunidade, como o PET- Saúde (MADRUGA et al., 2015).

Quanto à busca por Ligas Acadêmicas, pode-se remeter a uma tentativa de complementação de conteúdos não contemplados na matriz curricular, assim como outras atividades de extensão universitária, uma busca por espaços de integração (HAMAMOTOFILHO, 2011; SILVA; RIBEIRO, 2018).

Embora importantes e com significativa contribuição na formação dos estudantes de saúde, as atividades extensionistas configuram a maior oportunidade de visualização das práticas e do trabalho interprofissional (SILVA et al., 2021).

\section{Estímulo por parte da Universidade}

São unânimes os relatos sobre a falta de estímulo por parte da Universidade, a não ser os entrevistados que mencionaram as trocas das matrizes curriculares de alguns cursos que aconteceram a partir de 2015, inclusive do curso de Terapia Ocupacional, em 2016. Embora seja óbvio que a formação interprofissional não se efetive apenas com intenções ou textos, a troca deve acontecer desde a teoria à prática, com ações integradoras que permeiem pelas diversas profissões (SILVA; SCAPIN; BATISTA, 2011). 
Segundo os entrevistados, essa mudança vai propiciar que o aluno desde o primeiro ano tenha contato com a atuação interprofissional, podendo, dessa forma, compreender melhor esse tipo de atendimento.

Nessa perspectiva, os projetos de extensão e ligas acadêmicas foram mencionados como uma forma de incentivo por parte da Universidade, mas afirmado também que, acontecem de forma muito branda, onde na maioria das vezes o próprio aluno que busca se inserir nessas atividades e que a Universidade deveria incentivar um pouco mais.

Eu penso que não, é muito difícil a gente ter atividades até culturais mesmo dentro da universidade, que a gente possa compartilhar, até não só saber das questões do curso, mas conhecer, estar mais próximo, que isso também possibilita conhecer o que é do outro porque a gente tá mais junto e a gente não tem isso na universidade mesmo é déficit grande quanto a isso, por isso, não a gente não tem incentivo da universidade (Entrevistado 04).

Com relação ao estímulo por parte dos professores na formação do conhecimento integrado com outras áreas profissionais, os entrevistados divergiram um pouco. A maioria diz que não recebeu incentivo nos quatro primeiros anos de graduação, outros disseram ser estimulado apenas por alguns professores, principalmente nas áreas de Saúde Mental e Saúde Coletiva.

Esse fator pode estar relacionado às práticas e modelos de atuação próprios dessas áreas, com visão da saúde como fenômeno social, com abordagem interdisciplinar, que busca romper com os paradigmas e tendências que valorizam o olhar sobre a doença em detrimento da saúde como processo e expressão dos determinantes psicossociais, sócios dinâmicos e institucionais (SCARCELLI; ALENCAR, 2009).

Para contribuir com a formação interprofissional dentro da Universidade, os entrevistados propõem que ocorram mudanças nas matrizes curriculares como as que estão sendo feitas, com a nova proposta de integração entre os alunos, desde o primeiro ano de curso, onde os mesmos participam de módulos cujos eixos englobam vários cursos. 


\section{CONCLUSÃO}

O estudo evidenciou que nem todos os estudantes vivenciaram práticas interprofissionais e os que relataram alguma vivência nesse sentido, tiveram essa experiência no período próximo ao término do curso, no momento dos estágios, porém sem preparação teórica ou prática, tornando a experiência mais difícil.

De toda forma, na visão dos estudantes, ficou claro que a formação integrada possibilita grande aprendizado, mudança de paradigmas, maior conhecimento sobre outras profissões. Houve nítida carência de estímulos por parte da Universidade segundo os estudantes. Além disso, evidenciou-se que os professores poderiam incentivar mais a aprendizagem interprofissional. Outro aspecto importante é que a nova matriz curricular do curso de Terapia Ocupacional deve buscar preencher as lacunas na formação que os estudantes indicam, propiciando experiências integradoras antes da chegada ao estágio.

Outro aspecto que se destaca no estudo é que as atividades extracurriculares são importantes e necessárias, mas não deveriam ter a finalidade de suprir falhas apresentadas na formação integrada e que são evidenciadas ao longo do curso.

Considera-se importante que este estudo possa ser repetido ao longo dos próximos anos para que as mudanças propostas na nova matriz do curso sejam estudadas e ajustes sejam feitos se forem evidenciadas necessidades com relação à integração dos conhecimentos formativos e formação interprofissional.

\section{AGRADECIMENTO}

Conselho Nacional de Desenvolvimento Científico e Tecnológico (CNPq) e Fundação de Amparo à Pesquisa do Estado de Alagoas (FAPEAL) pelo financiamento através de Bolsas de Iniciação Científica (IC) que permitiram a realização desta pesquisa.

\section{REFERÊNCIAS BIBLIOGRÁFICAS}

ARAÚJO, T. A. M.; VASCONCELOS, A. C. C. P.; PESSOA, T. R. R. F.; FORTE, F. D. S. Multiprofissionalidade e interprofissionalidade em uma residência hospitalar: o olhar de residentes e preceptores. Interface (Botucatu), 
Botucatu, $\quad$ v. 21, n. 62, p. 601-613, Set. 2017. Disponível em: https://www.scielo.br/pdf/icse/v21n62/1807-5762-icse-1807-576220160295.pdf. Acesso 20 de Julho de 2019.

ARRUDA, G. M. M. S.; BARRETO, I. C. H. C.; RIBEIRO, K. G.; FROTA, A. C. O desenvolvimento da colaboração interprofissional em diferentes contextos de residência multiprofissional em Saúde da Família. Interface (Botucatu), Botucatu, v. 22, supl. 1, p. 1309-1323, 2018. Disponível em: https://www.scielo.br/pdf/icse/v22s1/1807-5762-icse-1807-576220160859.pdf. Acesso em: 20 de Julho de 2019.

BARDIN, L. Análise de conteúdo. Trad. Luís Antero Reto, Augusto Pinheiro. São Paulo: Edições 70, 2011.

CAMARGOS, M. A.; FERREIRA, M. V. F. B.; MAXTA, B. S. B.; PEDROSO TOMASI, A. R. P. A inserção dos terapeutas ocupacionais na rede de atenção à saúde do SUS em Minas Gerais entre os anos de 2005 e 2015. Cad. Bras. Ter. Ocup., São Carlos, v. 25, n. 2, p. 363-372, 2017. Disponível em: http://www.cadernosdeterapiaocupacional.ufscar.br/index.php/cadernos/article/view/1732. Acesso em: 20 de Julho de 2020.

CECCIM, R. B.; FEUERWERKER, L. C. M. O quadrilátero da formação para a área da saúde: ensino, gestão, atenção e controle social. Physis, Rio de Janeiro, v. 14, n. 1, p.41-65, 2004. Disponível em: http://www.scielo.br/scielo.php?script=sci_arttext\&pid=S0103-73312004000100004\&lng=en\&nrm=iso. Acesso em: 12 de setembro de 2019.

COLLISELLI, L.; TOMBINI, L. H. T.; LEBA. M. E.; REIBNITZ, K. S. Estágio curricular supervisionado: diversificando cenários e fortalecendo a interação ensino-serviço. Rev. bras. enferm., Brasília , v. 62, n. 6, p. 932-937, 2009. Disponível em: http://www.scielo.br/scielo.php?script=sci_arttext\&pid=S003471672009000600023\&lng=en\&nrm=iso. Acesso em: 12 de setembro de 2019.

DRUMMOND, A. F. Fundamentos da terapia ocupacional. In: CAVALCANTI, A.; GALVÃO, C. Terapia Ocupacional: fundamentação \& prática. Rio de Janeiro: Guanabara Koogan, 2007. p. 10-17. PMCid:PMC2474914.

FERREIRA, G. Formação e atuação interprofissional no Centro Pró Sorriso da Universidade de alfenas - UNIFENAS: Trabalho em equipe e educação interprofissional na atenção às deformidades: uma análise. 2017. 109 f. Dissertação (Mestrado profissional) - Universidade de São Paulo. São Paulo. 2017.

FURTADO, J. P. Equipes de referência: arranjo institucional para potencializar a colaboração entre disciplinas e profissões. Interface (Botucatu), Botucatu , v. 11, n. 22, p. 239-255, Ago. 2007. Disponível em: http://www.scielo.br/scielo.php?script=sci_arttext\&pid=S1414-32832007000200005\&lng=en\&nrm=iso. Acesso em: 25 de Maio de 2020.

HORSBURGH, M.; LAMDIN, R.; WILLIAMSON, E. Multiprofessional learning: the attitudes of medical, nursing and pharmacy students o shared learning. Medical Education, Oxford, GB, n. 35, p. 876-883, 2001. Disponível em: https://pubmed.ncbi.nlm.nih.gov/11555226/. Acesso em: 12 de Setembro de 2019.

LINS, S.R.A.; MATSUKURA, T.S. A formação graduada do terapeuta ocupacional no campo da saúde mental: a perspectiva de discentes e egressos. Cad. Ter. Ocup. UFSCar, São Carlos, v. 23, n. 4, p. 689-699, 2015. Disponível em: http://www.cadernosdeterapiaocupacional.ufscar.br/index.php/cadernos/article/view/1192. Acesso em: 20 de Julho de 2020.

MADRUGA. L. M. S.; RIBEIRO, K. S. Q. S.; FREITAS, C. H. S. M.; PÉREZ, I. A. B.; PESSOA, T. R. B. F.; BRITO, G. E. G. O PET-Saúde da Família e a formação de profissionais da saúde: a percepção de estudantes. Interface (Botucatu), Botucatu, $\quad$ v. 19, supl. 1, p. 805-816, 2015. Disponível em: http://www.scielo.br/scielo.php?script=sci_arttext\&pid=S1414-32832015000500805\&lng=en\&nrm=iso. Acesso em: 20 de Julho de 2019.

MEDEIROS, M. H. R. Terapia ocupacional: um enfoque epistemológico e social. São Carlos: Edufscar, 2009.

MINAYO M. C. S. O desafio do conhecimento: pesquisa qualitativa em Saúde. 12ª edição. São Paulo: Hucitec, 2010. 
MORETTI-PIRES, R. O. Complexidade em Saúde da Família e formação do futuro profissional de saúde. Interface (Botucatu), Botucatu, v. 13, n. 30, p. 153-166, 2009. Disponível em: http://www.scielo.br/scielo.php?script=sci_arttext\&pid=S1414-32832009000300013\&lng=en\&nrm=iso. Acesso em: 12 de Agosto de 2019.

PERES, C. M.; ANDRADE, A. S.; GARCIA, S. B. Atividades extracurriculares: multiplicidade e diferenciação necessárias ao currículo. Rev. bras. educ. med., Rio de Janeiro, v. 31, n. 3, p. 203-211, 2007. Disponível em: http://www.scielo.br/scielo.php?script=sci_arttext\&pid=S0100-55022007000300002\&lng=en\&nrm=iso. Acesso em: 21 de Janeiro de 2017.

SCARCELLI, I.; ALENCAR, S. Saúde Mental e Saúde Coletiva: intersetorialidade e participação em debate. Cadernos Brasileiros de Saúde Mental, v. 1, n¹, 2009.

SILVA, J. V. S.; RIBEIRO, M. C. O docente de Enfermagem e sua percepção sobre as ações integrativas na Saúde e na formação interprofissional. Revista Docência do Ensino Superior, v. 8, n. 2, p. 245-261, 2018. Disponível em: https://doi.org/10.35699/2237-5864.2018.2464. Acesso em: 14 de Agosto de 2020.

SILVA, J. V. S.; SANTOS JR, C. J.; RIBEIRO, M. C. Ensino integrado em Saúde e prática interprofissional. Revista Docência do Ensino Superior, v. 9, p. 1-14, 2019. Disponível em: https://doi.org/10.35699/2237-5864.2019.14820. Acesso em: 14 de Agosto de 2020.

SILVA, J. V. S.; SANTOS JR, C. J.; BRANDÃO, T. M.; RIBEIRO, M. C. Congresso Alagoano de Saúde Mental: experiências, desafios e contribuições para a formação na atenção psicossocial. Revista Docência do Ensino Superior, v. 11, p. 1-15, 2021. Disponível em: https://doi.org/10.35699/2237-5864.2021.19800. Acesso em 14 de Agosto de 2020.

SILVA, R. H. A.; SCAPIN, L. T.; BATISTA N. A. Avaliação da formação interprofissional no ensino superior em saúde: aspectos da colaboração e do trabalho em equipe. Campinas; Sorocaba, SP, v. 16, n. 1, p. 167-184, mar. 2011. Disponível em: https://www.scielo.br/pdf/aval/v16n1/v16n1a09.pdf. Acesso em: 14 de Agosto de 2020.

SOARES, L. B. T. História da terapia ocupacional. In: CAVALCANTI, A.; GALVÃO, C. Terapia ocupacional: fundamentação \& prática. Rio de Janeiro: Guanabara Koogan, 2007. p. 3-9. PMCid:PMC2474914.

TORRES, M. L. R. O brincar e suas implicações no cotidiano de crianças hospitalizadas: Relato de experiência. In: Congresso Brasileiro de Terapia Ocupacional - CBTO, 2015. Rev. Interinst. Bras. Ter. Ocup. Rio de Janeiro; ATOERJ, 2016.

UCHÔA, A. C.; VIEIRA, R. M. V.; ROCHA, P. M.; ROCHA, N. S. D.; MAROTO, R. M. Trabalho em equipe no contexto da reabilitação infantil. Physis, Rio de Janeiro, v. 22, n. 1, p. 385-400, 2012. Disponível em: http://www.scielo.br/scielo.php?script=sci_arttext\&pid=S0103-73312012000100021\&lng=en\&nrm=iso. Acesso em: 20 de Julho de 2019.

\section{(cc) Br}

Este trabalho está licenciado com uma Licença Creative Commons - Atribuição 4.0 Internacional. 